\title{
BOUNDED POINT DERIVATIONS AND REPRESENTING MEASURES ON $R(X)$
}

\author{
DONALD R. WILKEN
}

Let $A$ be a function algebra on $X$ and let $p$ be a point of $X$. A point derivation $D$ on $A$ at $p$ is a linear functional (not necessarily continuous) on $A$ satisfying

$$
D(f g)=D(f) g(p)+f(p) D(g), \quad \text { for all } f, g \in A .
$$

If $D$ is continuous, $D$ is called a bounded point derivation.

For a compact set $X$ in the complex plane $C$ we use $R_{0}(X)$ to denote the algebra of all rational functions having no poles on $X$. Denote by $R(X)$ the function algebra consisting of all functions which are uniform limits on $X$ of functions in $R_{0}(X)$.

If $p$ is a point of $X$ it is easy to see that there is a bounded point derivation at $p$ on $R(X)$ if and only if, for all $f$ in $R_{0}(X)$,

$$
\begin{gathered}
\left|f^{\prime}(p)\right| \leqq M\|f\| \quad \text { for some constant } M \\
(\|f\|=\sup \{|f(z)|: z \in X\}) .
\end{gathered}
$$

Recently Hallstrom [2], using the techniques of Melnikov [3], has given a very nice characterization, expressed in terms of analytic capacity, describing when points in $R(X)$ admit bounded point derivations. He also discussed the notion of point derivations of higher orders on $R(X)$. For a positive integer $k$, a bounded point derivation of order $k$ on $R(X)$ at $p$ is said to exist if there is a constant $M$ such that, for all $f$ in $R_{0}(X)$,

$$
\left|f^{(k)}(p)\right| \leqq M\|f\|,
$$

where $f^{(k)}(p)$ denotes the $k$ th derivative of $f$ at $p$. Hallstrom extended his characterization for bounded point derivations of order 1 to include those of order $k$.

In this note we obtain a similar characterization in terms of representing measures. That is, we show there exist bounded point derivations on $R(X)$ of order $k$ at a point $p$ if and only if $p$ has (complex) representing measures of a certain type. By a measure on $X$ we mean a finite complex Baire measure on $X$. If $\mu$ is a measure, $|\mu|$ denotes its total variation. We call $\mu$ a (complex) representing measure for a point $p$ in $X$ if

Received by the editors December 6, 1968 and, in revised form, August 5, 1969. 


$$
f(p)=\int f d \mu \quad \text { for every } f \text { in } R(X) .
$$

THEOREM. There exists a nonzero bounded point derivation on $R(X)$ of order $k \geqq 1$ at a point $p$ in $X$ if and only if there is a (complex) representing measure $\mu_{p}$ for $p$ satisfying

$$
\int d\left|\mu_{p}\right|(z) /|z-p|^{k}<\infty .
$$

Proof. Suppose there exists a nonzero bounded point derivation on $R(X)$ of order $k$ at $p$. Then, for all $f$ in $R_{0}(X)$,

$$
\left|f^{(k)}(p)\right| \leqq M\|f\| \quad \text { for some constant } M \text {. }
$$

Hence there exists a measure $\mu$ on $X$ with

$$
f^{(k)}(p)=\int f(z) d \mu(z), \quad f \in R_{0}(X) .
$$

Let $g(z)=(z-p)^{k} \in R_{0}(X)$. Then for each $f \in R_{0}(X)$,

$$
(f g)^{(k)}(p)=k ! f(p) .
$$

Thus

$$
f(p)=\frac{1}{k !} \int f(z)(z-p)^{k} d \mu(z)
$$

for all $f$ in $R_{0}(X)$. Letting $\mu_{p}=(1 / k !)(z-p)^{k} \mu$ and taking uniform limits, we obtain

$$
f(p)=\int f(z) d \mu_{p}(z)
$$

for all $f$ in $R(X)$. Clearly

$$
\int \frac{d\left|\mu_{p}\right|(z)}{|z-p|^{k}}<\infty,
$$

and we have the desired representing measure.

Conversely, suppose such a measure $\mu_{p}$ exists. Since $\mu_{p}$ is a representing measure it is an easy computation to show that no linear combination of the measures $\mu_{p},(z-p)^{-1} \mu_{p}, \cdots,(z-p)^{-k} \mu_{p}$ is orthogonal to $R(X)$-integration against an appropriate power of $z-p$ is nonzero. However, every $f \in R_{0}(X)$ satisfying $f(p)=f^{\prime}(p)=\cdots$ $=f^{(k)}(p)=0$ is annihilated by these measures. It follows that the 
closure of such functions has codimension $k+1$ in $R(X)$. Thus for each $j, 1 \leqq j \leqq k$, the functional $f \rightarrow f^{(j)}(p)$ must extend continuously to $R(X)$, as required.

In [4] Wermer constructed a compact set $X$ in $C$ for which $R(X)$ is proper in $C(X)$, but $R(X)$ admits no bounded point derivations. Using Wermer's example we find the following two corollaries surprising.

Corollary. There exists a compact set $X$ in $C$ with $R(X) \neq C(X)$ but no point of $X$ has a representing measure $\mu_{p}$ satisfying

$$
\int \frac{d \mu_{p}(z)}{|z-p|}<\infty .
$$

Corollary. There exists a compact set $X$ in $C$ with $R(X) \neq C(X)$ and with the following property: If $P$ is a nontrivial part of $X$ and $\left\{\mu_{q}\right\}$ is any family of representing measures in one-to-one correspondence with the points of $P$, then for each $p \in P,\left\{q \in P: \mu_{q}\right.$ is boundedly absolutely continuous with respect to $\left.\mu_{p}\right\}$ has zero planar Lebesgue measure.

Proof. Use Wermer's example together with the well-known fact that, for any measure $\mu$ with compact support,

$$
\int \frac{d|\mu|(z)}{|z-t|}<\infty
$$

almost everywhere in $t$ with respect to planar Lebesgue measure.

This example should be contrasted with the case where $X$ has non empty interior. For then the points in each component of int $X$ always have representing measures which are pairwise mutually boundedly absolutely continuous (see [1]).

\section{REFERENCES}

1. L. Bungart, Representing measures for algebras of holomorphic functions, Proc. Internat. Sympos. Function Algebras (Tulane Univ., 1965) Scott-Foresman, Chicago, IIl., 1966, pp. 153-156. MR $33 \# 1753$.

2. A. P. Hallstrom, On bounded point derivations and anlaytic capacity, Brown University Mathematics Department Publication, Providence, R. I., 1968.

3. M. S. Mel'nikov, Estimate of the Cauchy integral over an analytic curve, Mat. Sb. 71 (113) (1966), 503-514; English transl., Amer. Math. Soc. Transl. (2) 80 (1969), 243-255. MR 34 \#6120.

4. J. Wermer, Bounded point derivations on certain Banach algebras, J. Functional Analysis 1 (1967), 28-36. MR 35 \#5948.

State University of New York at Albany 